\title{
Enhancing Long-term Children to Robot Interaction Engagement through Cloud Connectivity
}

\author{
Jordi Albo-Canals \\ CEEO - Tufts University \\ 200 Boston Ave, 02155 \\ Medford MA, US \\ Jordi.Albo_Canals@tufts.edu
}

\author{
Adso Fernández-Baena, \\ Roger Boldu, Alex Barco, \\ Joan Navarro, David Miralles \\ La Salle BCN - URL \\ Quatre Camins, 08022 \\ Barcelona, Spain \\ adso@salleurl.edu
}

\author{
Cristobal Raya, Cecilio Angulo \\ GREC-UPC \\ 1 Victor Balaguer Av, 08800 \\ Vilanova i la Geltrú, Spain \\ cristobal.raya@upc.edu
}

\begin{abstract}
In this paper, we introduce a cloud-based structure to enhance long-term engagement in a pet-robot companion treatment to reduce stress an anxiety to hospitalized children. Cloud connectivity enables to combine human intervention with artificial intelligent multi-agent to bias the Robot Companion behavior in order to foster a better engagement and decrease the drop out during the treatment.
\end{abstract}

\section{Categories and Subject Descriptors}

I.2.9 [Robotics] : Commercial robots and applications. D.2.2. [Design Tools and Techniques]: User interfaces. H.1.2 [User/Machine Systems] : Human factors, Human information processing.

\section{General Terms}

Management, Measurement, Performance, Design, Reliability, Experimentation, Security, Human Factors, Verification

\section{Keywords}

Robotics; Robot; Play; Children; Engagement; Long-term

\section{INTRODUCTION}

Therapy with animal pets has been proved to be successful in several situations [1]. Children tend to develop engagement, empathy, and enjoyment feelings with their animal pets [2]. These feelings reduce their stress and anxiety.

Pet like robots are an alternative to the real pets when improving patient experience at hospitals by being a distractor that reduces anxiety in situations such as before surgery or painful treatments [3]. In [4] a study is presented as well as a comparison of different commercial pet-robot animals to describe the relevant characteristics to foster a useful interaction between pet robots and humans. One of the key parameters to measure identified in [4] is the engagement.

The challenge to increase the engagement between the robot and the child is based on the fundamental that of master-pet bond may emerge between a child and a pet robot with social skills, and it implies a hierarchy between master and pet that could be

Permission to make digital or hard copies of part or all of this work for personal or classroom use is granted without fee provided that copies are not made or distributed for profit or commercial advantage and that copies bear this notice and the full citation on the first page. Copyrights for third-party components of this work must be honored. For all other uses, contact the Owner/Author. Copyright is held by the owner/author(s).

HRI'15 Extended Abstracts, March 2-5, 2015, Portland, OR, USA. ACM 978-1-4503-3318-4/15/03.

http://dx.doi.org/10.1145/2701973.2702045 improved if the robot has a positive response to a master demand during the long-term interaction [5].

The robot used in this study is the PLEO rb robot. PLEO is a lowcost baby Camarasaurus robot with a bio-inspired behavior that captures attention of the children. However, as every child reacts differently to the same robot stimulus, we establish the hypothesis that inducing a supervised behavioral state to the robot we can enhance the engagement. Through the behavior induction we obtain a customized response of the robot that is proved to reduce the drop out in the human robot interaction [6].

In this paper we introduce a cloud architecture for the coexistence of multiple systems that allows either the end user, a human supervisor, or an artificial agent to induce PLEO behavioral states. In the following we define: 1) the PLEO behavioral parameters that can enhance the engagement, 2) technical set up: how PLEO works and how we can act over the robot, the cloud layout, and how users can contribute to stimulate the PLEO, and 3) preliminary results of test sessions in oncology rooms, waiting rooms, and pre-surgery rooms.

\section{PLEO BEHAVIORAL PARAMETERS}

The PLEO robot is endowed with a Life OS system that allows the robot to evolve through time and to interact in a bio-inspired way. It is an event-based behavior that plays its implemented micro behaviors like a role game character according to the experience, the acquired skills, and a set of parameters that change over time. To identify the key PLEO behavioral parameters we ran sessions using the robot in a regular school with 20 kids playing with the PLEO in pairs, in different hospitals facilities in one-to-one kid-PLEO interaction and in group sessions with multiple kids and multiple PLEOs at the same time, and in a robotics summer camp with kids who already saw the PLEO robot in the past. Before and after each activity we measured the internal variables of the PLEO robot. Specifically, these variables are: Mood, Physical, Emotion, Health, Feed, Activity, Obedience, and Skill. All of them are in a percentage scale. Values for these variables affect the PLEO's response when interacting with kids. For instance, if the Feed is at $100 \%$, the PLEO will refuse to eat, or Obedience close to $0 \%$ means that the PLEO is not following orders. As a real pet, if these parameters are not modified, the interaction can drive into frustration and decrease engagement. Through the observation, we identify the Physical, Feed, Activity, and Obedience as the key factors that play is significant role in the drop out of kids during the play game with the robot.

\section{TECHNICAL SET UP}

The technical set up implies the modification of the PLEO robot to expand its connectivity, the implementation of a scalable cloud 
layout to manage the data transfer between the cloud agents, and different interactive devices to get better engagement.

\subsection{The Improved PLEO rb}

To transform a toy robot like PLEO to a cloud system client we need to expand its connectivity minimizing the intervention over the hardware of the system, without modifying its pre-programed bio-inspired behavior, without modifying its embodiment, and keeping a reliable trade-off between power consumption, data transfer, and connectivity to the cloud. Thus, we aimed to implement a new battery system with an embedded Bluetooth antenna that links up to multiple PLEOs to a Raspberry Pi based hub/router.

\subsection{The Cloud Concept}

The cloud maximizes the effectiveness of the shared resources in a set of devices connected to a network. What we present here is a cloud architecture that allows users and artificial agents to induce behavioral states to a cloud composed of PLEO robot.

Figure 1 depicts a global overview of the project deployed in a private network with a local server on a computer. The local server runs over AMPPS and uses a Linksys N750 router. The treatment to the kids in different places is done with the Pleo rb whose behavior is biased through the cloud and its interactive devices.

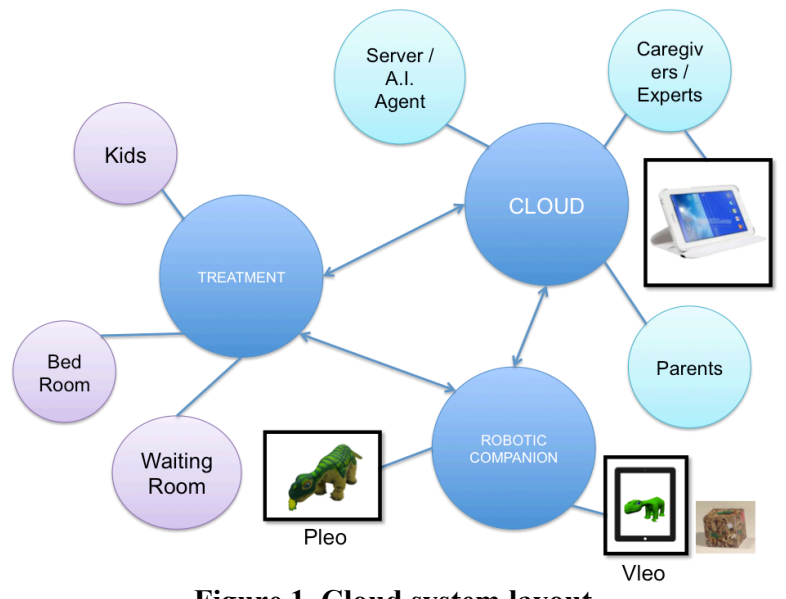

Figure 1. Cloud system layout

\subsection{Stimulation Devices}

The stimulation devices are those that modify the PLEO internal variables to create the most suitable context to keep the attention of the kids and consequently the engagement. These devices are:

1. The Artificial Intelligent Agent on the server aimed to learn which decision parameters and descriptors are relevant.

1. A stimulation interface to allow caregivers, experts, or parents to induce a customized behavior to the PLEO changing the parameters listed in section 2 .

2. The VLEO, a Virtual Avatar that reproduces the PLEO and that interacts with the real PLEOs connected to the cloud. VLEO is controllable through a tangible user interface (TUI) and it is visualized thanks to augmented reality (AR). Thus, kids can see VLEO through a tablet screen, a virtual PLEO that is represented in the physical world by a cube while at the same time acts as a TUI and an AR marker. The cube leads children to control VLEO by gesturing with it. Each cube face has a different PLEO behavior configuration and evokes immediate responses from the PLEO. In Figure 2 we can see how it looks like.

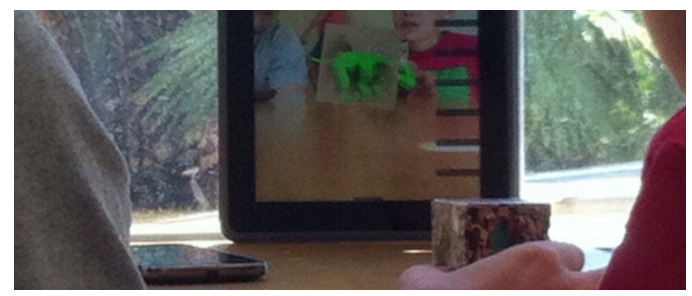

Figure 2. The VLEO and the cubic TUI interface

\section{RESULTS AND CONCLUSIONS}

During the regular school sessions all kids try to feed the PLEO, $34 \%$ of them forced the PLEO to eat. All of them prefer the PLEO to be moving most of the time, but $8 \%$ would like PLEO less active while they were pick it up. Low obedience level is correlated with kids' complaint about the PLEO, and low activity with lack of touching the robot. Also $100 \%$ of the caregivers would like to set up a customized initial state of the parameters before delivering the robots. They agreed that changing its parameters during the intervention can help keeping participants' attention.

8 over 10 interviewed kids after PLEO + VLEO interaction answered that VLEO highly contributes to the enjoyment.

The benefits observed during the sessions with PLEO are: To promote the kids' motor skills, alternative to a dog therapy, to help in cognitive therapy creating specific activities, and parents much happier to see their kids have a good time with PLEO. By enhancing the engagement we ensure better benefits during treatments with the robot.

\section{ACKNOWLEDGMENTS}

Authors thank Spanish Ministry of Economy and Competitiveness for its support under grant TIN2012-38416-C03-01,02

\section{REFERENCES}

[1] J. Gagnon et al. (2004), Implementing a hospital-based animal therapy program for children with cancer: a descriptive study, Canadian Oncology Nursing JournalRevue Canadienne de Nursing Oncologique, 14, 4.

[2] M. A. Halm (2008), The healing power of the human-animal connection, American Journal of Critical Care, 17, 4.

[3] J. Navarro et al. "A Cloud robotics architecture to foster individual child partnership in medical facilities." Cloud Robotics Workshop in 26th IEEE/RSJ Intl. Conference on Intelligent Robots and Systems, (2013).

[4] Heerink, Marcel, et al. "Exploring Requirements and Alternative Pet Robots for Robot Assisted Therapy with Older Adults with Dementia." Social Robotics. Springer International Publishing, 2013. 104-115.

[5] I. Leite, C. Martinho, and A. Paiva, "Social robots for longterm interaction: a survey," Intl Journal of Social Robotics, vol. 5, no. 2, pp. 291-308, 2013.

[6] A. Barco et al. "A drop-out rate in a long-term cognitive rehabilitation program through robotics aimed at children with TBI." RO-MAN: The 23rd IEEE Intl. Symposium on. Robot and Human Interactive Communication, IEEE, 2014. 